\title{
Characterization of self-heating in cryogenic high electron mobility transistors using Schottky thermometry
}

\author{
Alexander Y. Choi (D) ${ }^{1}$ Iretomiwa Esho (D) ${ }^{1}$ Bekari \\ Gabritchidze (D) ${ }^{2}$ Jacob Kooi (D), ${ }^{3}$ and Austin J. Minnich (D) ${ }^{1, *}$ \\ ${ }^{1}$ Division of Engineering and Applied Science, \\ California Institute of Technology, Pasadena, CA 91125, USA \\ ${ }^{2}$ Division of Physics, Mathematics, and Astronomy, \\ California Institute of Technology, Pasadena, CA 91125, USA \\ ${ }^{3}$ NASA Jet Propulsion Laboratory, California Institute of Technology, Pasadena, CA 91109, USA
}

(Dated: May 26, 2021)

\begin{abstract}
Cryogenic low noise amplifiers based on high electron mobility transistors (HEMTs) are widely used in applications such as radio astronomy, deep space communications, and quantum computing, and the physical mechanisms governing the microwave noise figure are therefore of practical interest. In particular, the contribution of thermal noise from the gate at cryogenic temperatures remains unclear owing to a lack of experimental measurements of thermal resistance under these conditions. Here, we report measurements of gate junction temperature and thermal resistance in a HEMT at cryogenic and room temperatures using a Schottky thermometry method. At temperatures $\sim 20 \mathrm{~K}$, we observe a nonlinear trend of thermal resistance versus power that is consistent with heat dissipation by phonon radiation. Based on this finding, we consider heat transport by phonon radiation at the low-noise bias and liquid helium temperatures and estimate that the thermal noise from the gate is several times larger than previously assumed owing to self-heating. We conclude that without improvements in thermal management, self-heating results in a practical lower limit for microwave noise figure of HEMTs at cryogenic temperatures.
\end{abstract}

\footnotetext{
* Corresponding author: aminnich@caltech.edu
} 


\section{INTRODUCTION}

Microwave low noise amplifiers (LNAs) based on high electron mobility transistors (HEMTs) are widely-used components of scientific instrumentation in fields such as radio astronomy [1, 2], deep space communication [3], and quantum computing [4-8]. After decades of development [9-13], HEMT LNAs have achieved cryogenic noise temperatures approximately 5-10 times the quantum limit over frequencies from 1-100 GHz [1]. Despite this progress, applications drive the development of amplifiers with ever-lower noise figures.

Noise in HEMT amplifiers is typically interpreted using the Pospieszalski model [14]. In this model, noise is decomposed into hot electron noise added in the channel and thermal noise in the gate, parameterized by equivalent temperatures $T_{d}$ and $T_{g}$, respectively. The gate noise temperature is typically assumed to be the cryostat base temperature $T_{g}=T$ while the drain temperature is fit to measured data. For a constant drain current, the hot electron contribution is taken to be constant and the minimum noise figure then scales as $T_{g}^{1 / 2}[2]$.

Although the noise temperature does decrease with base temperature over a range of temperatures as predicted, at liquid helium temperatures the noise temperature is observed to plateau to a value several times the quantum noise limit [15-17]. This noise temperature plateau has been attributed to a variety of mechanisms, including drain shot noise [18], gate leakage current [9], and self-heating [15, 19]. In particular, Ref. [15] used measurements of microwave noise to conclude that the thermal resistance associated with phonon radiation leads to non-negligible self-heating at cryogenic temperatures. However, this conclusion is based on an indirect estimate of the gate junction temperature using a noise model.

Measurements of the gate temperature under bias at cryogenic temperatures would provide more direct evidence that self-heating is the origin of the noise temperature plateau. This measurement is challenging for conventional thermometery techniques such as IR microscopy [20, 21], microRaman spectroscopy [22-24], or liquid crystal thermography [25] due to geometrical constraints like the sub-micron gate lengths and the buried structure of modern HEMTs. Consequently, selfheating in FETs is usually characterized with measurements of temperature-sensitive electrical parameters. Early semi-quantitative studies of self-heating in CMOS estimated the temperature under bias using the temperature-dependence of drain current [26-29]. However, these approaches neglected a number of mechanisms relevant to the drain current in sub-micron devices such as the bias dependence of threshold voltage, series resistances, and non-stationary transport effects, which are known to be important in modern HEMTs and could affect the extracted temperature 
rise. Later studies of self-heating in MOSFETs incorporated some of these effects and reported measurements of temperature rise and thermal time constants [30]. Recent work in SOI MOSFETs reported that the dominant thermal resistance is due to the buried oxide layer [31]. Self-heating studies in HEMTs have largely focused on GaN power FETs at room temperature, where device lifetime is limited by channel heating [32]. Typically, the temperature rise is extracted from pulsed current measurements on the gate $[33,34]$, but this technique is generally unsuitable for cryogenic HEMTs where the thermal time constants are on the same order as the pulse duration [35]. As a result, self-heating in cryogenic III-V HEMTs remains poorly characterized.

Here, we present measurements of the junction temperature and thermal resistance of the metallic gate in low-noise GaAs HEMTs using Schottky thermometry. At cryogenic temperatures, we observe a nonlinear trend of the thermal resistance on dissipated power that is consistent with heat transport by phonon radiation. We analyze the implications of this finding at the low-noise bias in which the drain and gate are forward and reverse biased, respectively, using a radiative circuit model. The model predicts that the gate self-heats to a value comparable to the physical temperature of the drain, contradicting the typical assumption that the gate is isothermal with the base temperature. Our study thus implies that without improvements to device thermal management to remove heat from the gate, self-heating results in a practical lower limit for HEMT microwave noise figure at cryogenic temperatures.

\section{EXPERIMENT}

We measured the temperature of the gate-barrier junction of a discrete HEMT under DC bias using the Schottky thermometry method introduced in Ref. [36]. In brief, the method exploits the temperature dependence of the electrical parameters of the Schottky junction to infer the temperature rise of the junction using DC $I-V$ characteristics and microwave $S$ parameters. The current in a Schottky diode is given by:

$$
I=I_{S} \exp \left(\frac{q\left(V-I R_{S}\right)}{\eta k_{B} T_{j}}\right)
$$

where $q$ is the elementary charge, $k_{B}$ is the Boltzmann constant, $R_{S}$ is the parasitic series resistance, $I_{S}$ is the saturation current, $\eta$ is the ideality factor, and $T_{j}$ is the intrinsic junction temperature.

To obtain $T_{j}$ for a given bias, the temperature dependence of $I_{S}$ and $\eta$ is extracted from DC $I$ - $V$ 
characteristics by varying the cryostat base temperature at low bias where self-heating is negligible. Next, the small-signal resistance about a DC bias is determined from the microwave $S$ parameters. Equating expressions for the series resistance yields an equation including the measured Schottky and $S$ Parameters in which the only unknown is the junction temperature:

$$
\frac{V}{I}-\frac{\eta\left(T_{j}\right) k_{B} T_{j}}{q I} \ln \left(\frac{I}{I_{S}\left(T_{j}\right)}\right)=r_{T}-\frac{\eta\left(T_{j}\right) k_{B} T_{j}}{q I}
$$

Here, $r_{T}$ is the small signal resistance obtained from the $\mathrm{S}$ parameters. With the DC $I-V$ characteristics and S parameters for different biases known, we obtain the junction temperature at various base temperatures by numerically solving Eqn. 2 .

We used this method to characterize the thermal resistance of a $70 \mathrm{~nm}$ gate length InAlAs/InGaAs mHEMT with a $200 \mu \mathrm{m}$ gate width. Further details of the device are specified in Ch. 5.1 of Ref. [37]. All measurements were performed in a custom cryogenic probe station [38] with cryostat base temperature (denoted base temperature, $T$ ) controlled between $20 \mathrm{~K}$ and $300 \mathrm{~K}$ by a LakeShore 336 temperature controller. The HEMT was biased using a Minicircuits ZX85-12G-S+ bias-tee. S-parameter measurements were performed with a Rohde \& Schwarz ZVA50 vector network analyzer from $10 \mathrm{MHz}$ to $18 \mathrm{GHz}$, calibrated with the through-reflectmatch method. The DC measurements were corrected for loss in the bias-tee and coaxial lines. The temperature-dependence of the Schottky parameters $I_{s}$ and $\eta$ were extracted in the log-linear region at low bias $I \sim 100 \mu \mathrm{A}$ so that self-heating can be neglected but at sufficiently high bias so that the characteristic is still log-linear [39]. The saturation current and ideality factor were fit to the expressions derived from thermionic emission theory (See Eqns. 2 and 3 in Ref. [36]). The saturation current expression was modified by adding a temperature-independent term to Eqn. 3 in Ref. [36] to account for the leakage current that dominates at cryogenic temperatures. After extracting the junction temperature, we verified that the self-heating can be neglected in the fit range. The junction temperature was extracted at biases ranging from 0.5 to $10 \mathrm{~mA}$ for which the capacitance of the channel depletion layer is negligible. We restrict the biases to this range to avoid non-negligible corrections associated with removing parasitic capacitance. For the calculation of the thermal resistance, we took the area of the gate to be $14 \mu^{2}=70 \mathrm{~nm} \times 200 \mu \mathrm{m}$. 

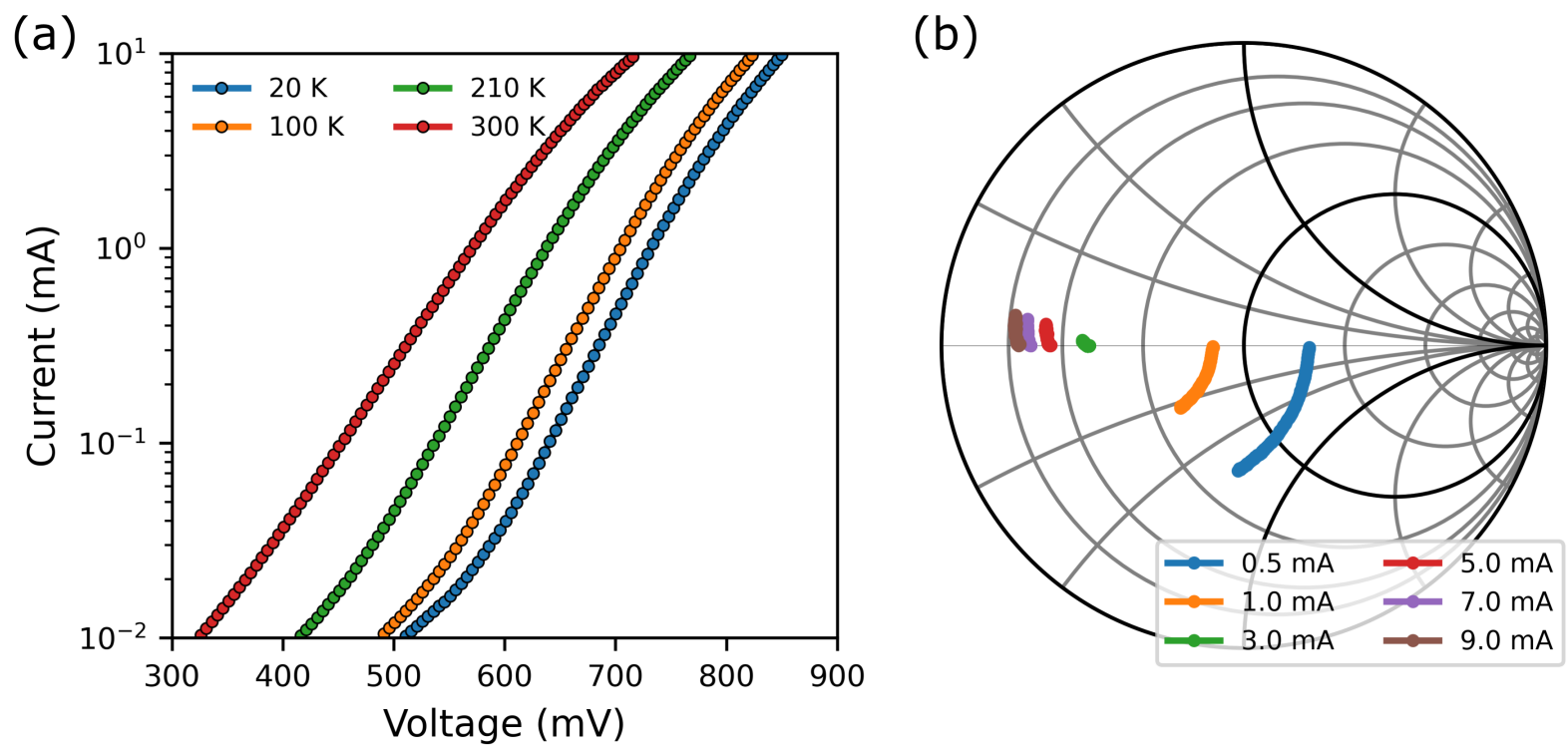

Figure 1: (a) Measured forward $I-V$ characteristics of the HEMT at different base temperatures (colored symbols). We fit the temperature-dependence of the Schottky parameters for $I \lesssim 100 \mu \mathrm{A}$ for which $T_{j} \approx T$ and the diode characteristics are log-linear. The DC resistance is combined with $S$ parameter measurements to extract the junction temperature at the $0.5-9 \mathrm{~mA}$ biases for which self-heating occurs. (b) Small-signal reflection coefficient extracted from high frequency measurements at different bias points (colored markers) and $T=20 \mathrm{~K}$ from 1-10 GHz. The small-signal resistance is extracted by taking the real part of the input impedance.

\section{RESULTS}

\section{A. Schottky $I-V$ measurements}

Figure 1a shows the measured forward bias DC $I-V$ characteristics at different base temperatures. As the device is cooled, the characteristics shift to higher threshold voltages. The DC data were used to extract the temperature dependence of the saturation current and ideality factor as discussed in Section II. Figure 1b shows the measured reflection coefficient $S_{11}$ at various bias points at $20 \mathrm{~K}$ from 1-10 GHz. The total small signal resistance is obtained from the reflection coefficient through $r_{T}=\operatorname{Re}\left[{ }^{6}\left(1+S_{11}\right) /\left(1-S_{11}\right)\right]$ where $Z_{0}$ is the characteristic impedance of the system.

Qualitative evidence of self-heating can be obtained by inspecting the $I-V$ characteristics. Ideal 
Schottky diodes exhibit log-linear $I-V$ characteristics, but in practice deviations are observed owing to self-heating and parasitic series resistance. Series resistance leads to a sublinear log $I-V$ while self-heating causes a superlinear trend (see Figure 3 in Ref. [36]). These effects depend on bias as well as temperature, and their balance determines the trend of the measured $I-V$ characteristic.
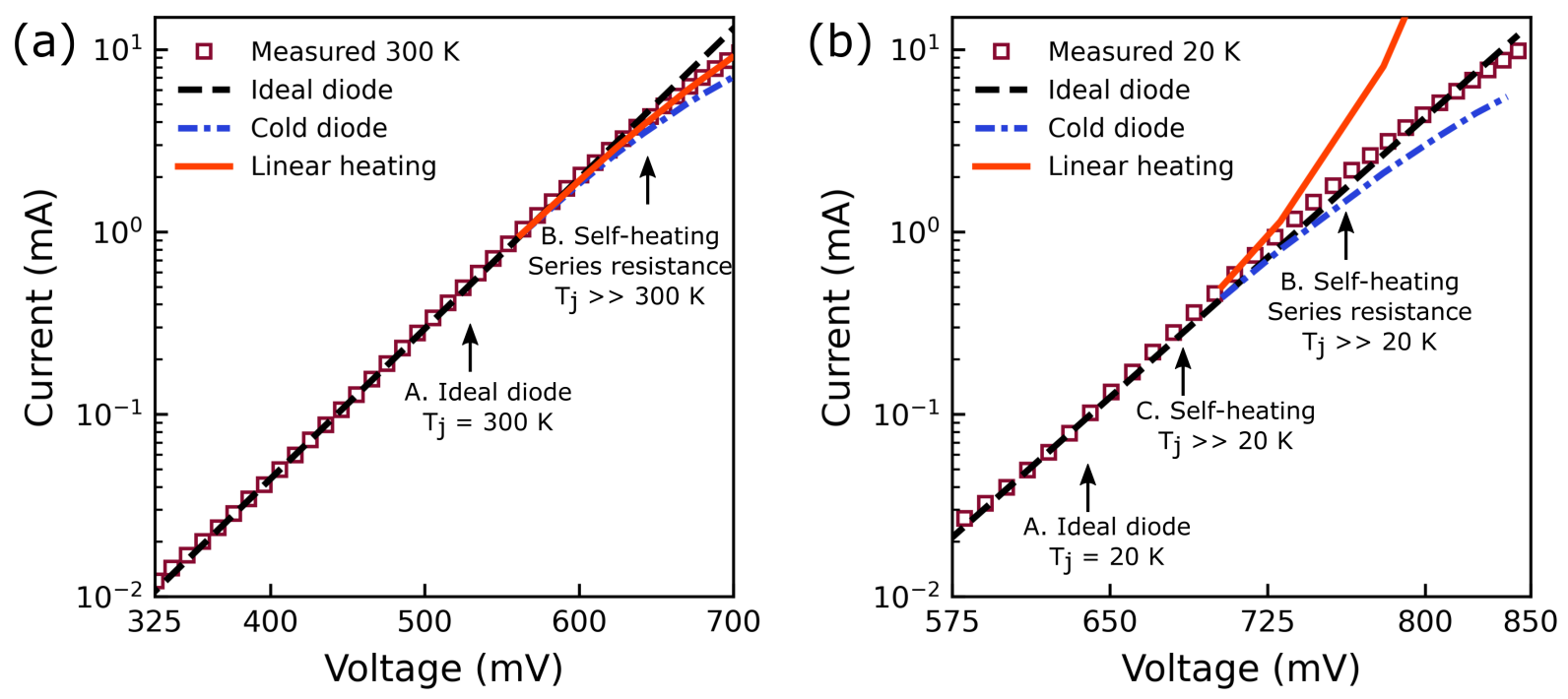

Figure 2: Measured $I-V$ characteristics at (a) $300 \mathrm{~K}$ and (b) $20 \mathrm{~K}$ (red markers) compared to the ideal diode (black dashed line), cold diode (blue dashed dotted line), and linear-heating model (orange solid line). A calculation assuming constant thermal resistance explains the measurements at $300 \mathrm{~K}$ but not at $20 \mathrm{~K}$. See text for detail. The curves for cold diode model and linear heating model coincide with the ideal diode model below $0.5 \mathrm{~mA}$ and are omitted for clarity.

Figure 2 compares the measured $I-V$ characteristics with those generated by an ideal Schottky model, a cold-diode model, and a model including self-heating with a constant thermal resistance $R_{t h}$. The ideal Schottky model neglects series resistance and self-heating and consequently exhibits log-linear DC characteristics at all biases. The cold-diode model incorporates the measured diode series resistance but neglects self-heating so that the junction temperature equals the base temperature at all biases, $T_{j}=T$. Finally, the linear-heating model incorporates series resistance, temperature-dependent Schottky parameters, and assumes that the junction temperature increases linearly with the dissipated power, $T_{j}=T+R_{t h} I V$, with thermal resistance $R_{t h}$ taken as a fitting parameter. Through comparison to the measured DC $I$ - $V$, we can infer the relative magnitude 
and power dependence of the junction temperature $T_{j}$ at different $T$.

Figure $2 \mathrm{a}$ shows the model comparison to measurements at $T=300 \mathrm{~K}$. At low biases below $1 \mathrm{~mA}$ the measured diode is nearly ideal and exhibits the expected log-linear trend (Region A). At $1 \mathrm{~mA}$, the cold-diode model, linear-heating model, and measured current agree to within $3 \%$, indicating that the temperature rise at this bias is small compared to the base temperature. At high currents exceeding $1 \mathrm{~mA}$, the series resistance leads to a sublinear trend (Region B); however, the cold-diode model including only series resistance underpredicts the measured current at high biases $(\sim 9 \mathrm{~mA})$ by $\sim 25 \%$. In contrast, the linear-heating model agrees with the measured $I-V$ characteristics in Region B. From this comparison, we infer that at $300 \mathrm{~K}$, self-heating is appreciable above $1 \mathrm{~mA}$ biases and that the thermal resistance is constant with power.

Figure $2 \mathrm{~b}$ shows the model comparison at $T=20 \mathrm{~K}$. As at $300 \mathrm{~K}$, below $100 \mu \mathrm{A}$ the diode is nearly ideal, but a super-linear trend associated with self-heating is evident at intermediate biases between $100 \mu \mathrm{A}$ and $1 \mathrm{~mA}$ (Region $\mathrm{C}$ ), which is evident observed in the room temperature characteristics. At $1 \mathrm{~mA}$, the measured current exceeds the cold-diode model by over 15\%, indicating that the temperature increase due to self-heating is substantially larger at this bias than at $300 \mathrm{~K}$. Furthermore, above $1 \mathrm{~mA}$ (Region B) the linear-heating model at $20 \mathrm{~K}$ markedly overpredicts the measured current, indicating that the thermal resistance must decrease as the bias increases at 20 K.

\section{B. Junction temperature extraction}

We now perform a quantitative analysis of the data by using the method in Sec. II to extract the junction temperature. Figure 3a shows the extracted junction temperature rise versus power for $T=300 \mathrm{~K}, 60 \mathrm{~K}, 40 \mathrm{~K}$ and $20 \mathrm{~K}$. The features of the temperature rise are consistent with the qualitative expectations developed in Fig. 2. First, at $T=300 \mathrm{~K}$, the temperature rise is nearly linear with the dissipated power, indicating that a constant thermal resistance can account for the measurements. Second, at the low bias point of $1 \mathrm{~mA}$ and $40 \mu \mathrm{W} \mathrm{\mu m}{ }^{-2}$, the junction temperature rise is $3 \mathrm{~K}$ or $1 \%$ of the base temperature, confirming the qualitative prediction of small temperature rise at this power shown in Fig. 2a. In contrast, at cryogenic temperatures, the temperature rise exhibits a nonlinear trend with power, with the temperature initially increasing rapidly but transitioning to a weaker increase at higher powers. This observation is consistent with Fig. $2 \mathrm{~b}$ and suggests that the thermal resistance decreases as the bias is increased. At $T=20$ $\mathrm{K}$ and the same low bias point of $1 \mathrm{~mA}$ and $50 \mu \mathrm{W} \mathrm{\mu m}{ }^{-2}$, the temperature rise is $29 \mathrm{~K}$, almost 

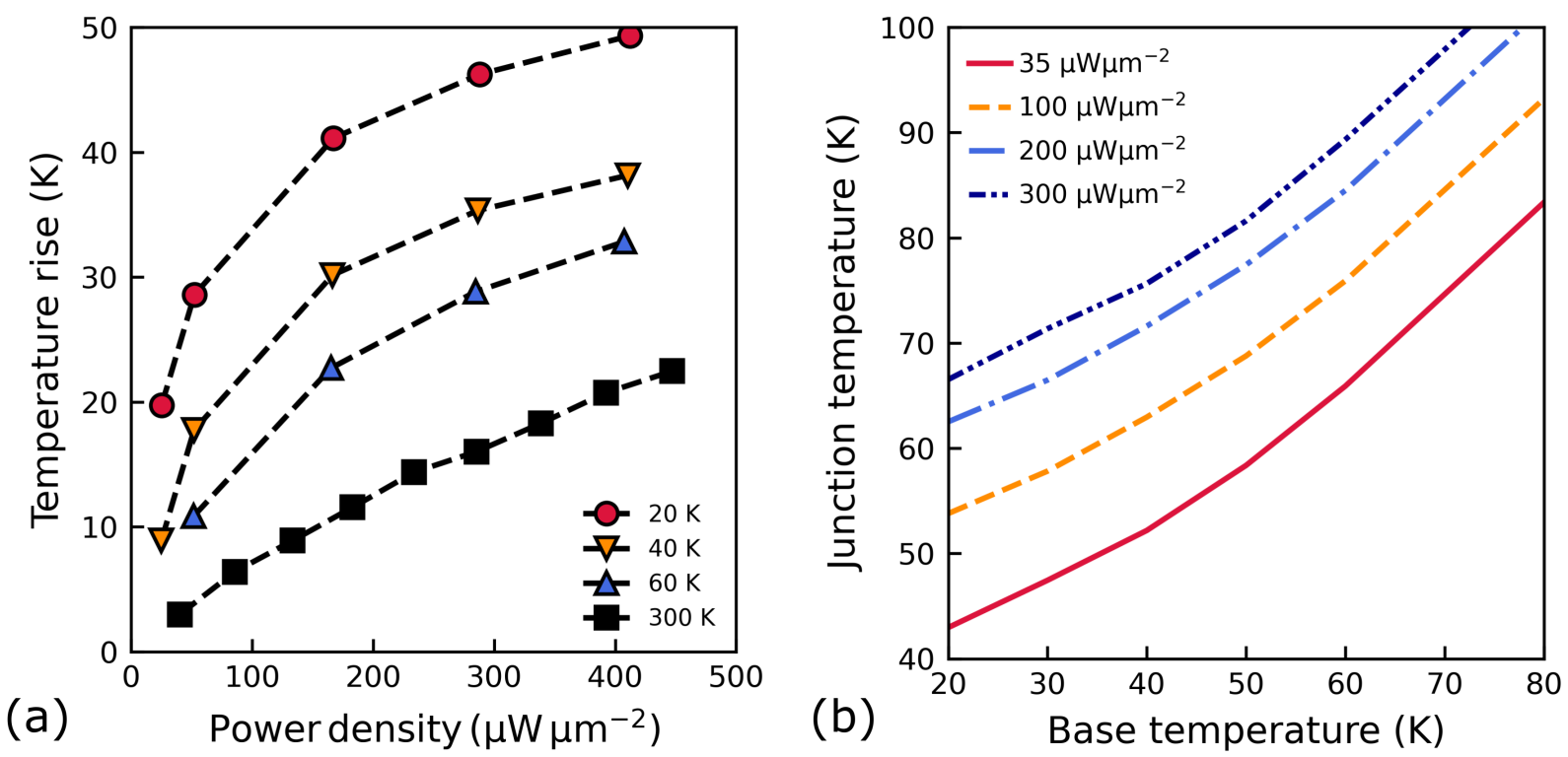

Figure 3: (a) Junction temperature rise, $T_{j}-T$, versus dissipated power density at base temperatures $20 \mathrm{~K}$ (red circles), $40 \mathrm{~K}$ (yellow triangles), $60 \mathrm{~K}$ (blue triangles), and $300 \mathrm{~K}$ (black squares). The temperature rise is approximately linear with power at room temperature but nonlinear at cryogenic temperatures. Dashed black lines are added as guides to the eye.

(b) Interpolated junction temperature versus base temperatures at various power densities (colored lines). As the device is cooled, the junction temperature begins to plateau due to self-heating.

10 times larger than the room temperature value of $3 \mathrm{~K}$. This difference is on the same order as the difference in heat capacity between these temperatures, which decreases by over an order of magnitude from $300 \mathrm{~K}$ to $20 \mathrm{~K}$ [40]. At $T=40 \mathrm{~K}$ and $60 \mathrm{~K}$, the temperature rise exhibits the same qualitative features as those seen at $20 \mathrm{~K}$, but for the same power, the temperature rise is smaller at higher base temperatures.

As described in Sec. II, the temperature is extracted for fixed gate current values. To extract the temperature dependence of the junction temperature at fixed power, we linearly interpolate the junction temperatures as a function of power. This procedure is analogous to taking a vertical slice at fixed power in Fig. 3a. Figure 3b shows the interpolated junction temperature versus the base temperature for various power densities applied to the gate. At base temperatures near 80 $\mathrm{K}$ and all powers, the junction temperature decreases with base temperature as $\Delta T_{j} / \Delta T \sim 0.9$ $\mathrm{K} / \mathrm{K}$, meaning that at these temperatures, the junction and cryostat are cooled at nearly the 
same rate. As the cryostat is cooled to $20 \mathrm{~K}$, the rate drops to $\sim 0.45 \mathrm{~K} / \mathrm{K}$, indicating that while the device continues to cool, the gate temperature cools less rapidly due to self-heating. The observed temperature saturation implies a nonlinear increase of the thermal resistance as the junction temperature approaches $\sim 50 \mathrm{~K}$.

We now compute the thermal resistance as the ratio of the junction temperature rise in Fig. 3a and the power density. Figure 4 shows the thermal resistance versus power density. At room temperature, the thermal resistance is nearly constant with power, as expected from Figs. 2a and 3a. As the device is cooled to cryogenic temperatures, the thermal resistance increases at all powers. At $1 \mathrm{~mA}$ and $40 \mu \mathrm{W} \mathrm{\mu m}{ }^{-2}$, the thermal resistance increases by almost an order of magnitude from $300 \mathrm{~K}$ to $20 \mathrm{~K}$. Furthermore, at cryogenic temperatures the thermal resistance exhibits a nonlinear power dependence.

\section{Phonon radiation resistance}

We now examine the magnitude of the measured thermal resistance in context of values reported for various metal-semiconductor interfaces. The HEMT gate is formed by depositing a metallic stack consisting of metals such as $\mathrm{Pt}, \mathrm{Ti}$, and $\mathrm{Au}$ on the InAlAs barrier layer that has been subjected to semiconductor processing steps such as wet etching. At $300 \mathrm{~K}$, reported values of thermal boundary conductance for a soft metal such as gold on semiconductor are in the range of 30-40 $\mathrm{MW} \mathrm{m}^{-2} \mathrm{~K}^{-1}$ [41]. These studies utilize pristine interfaces for which the metal is evaporated directly onto a high-quality crystalline substrate. In contrast, the etching step in the fabrication of the gate leaves an amorphous region several nm thick at the gate-semiconductor junction (see Figure 4.11(a) in Ref. [42]). Prior measurements report that crystalline disorder can suppress the thermal conductance and enhance thermal boundary resistance by factors of approximately 3-4 (see Figure 7 in Ref. [43]) as phonons with atomic-scale wavelengths are reflected at the interface [44]. At $300 \mathrm{~K}$, the average thermal resistance of the HEMT over all power levels is $60 \mathrm{~K} \mathrm{\mu m}^{2} \mathrm{~mW}^{-1}$. This value corresponds to a conductance of $17 \mathrm{MW} \mathrm{m}^{-2} \mathrm{~K}^{-1}$, which is consistent with the above values for thermal conductance of a defective interface.

We next examine the origin of the nonlinear trend of thermal resistance versus power in Fig. 4. Assuming the total gate thermal resistance is dominated by the thermal boundary resistance, in principle a microscopic model of thermal boundary resistance could be constructed from thermal resistance versus temperature and knowledge of the phonon density of states of the semiconductor and metal. However in practice, the gate is formed via deposition of alternating layers of $\mathrm{Ti}$, 


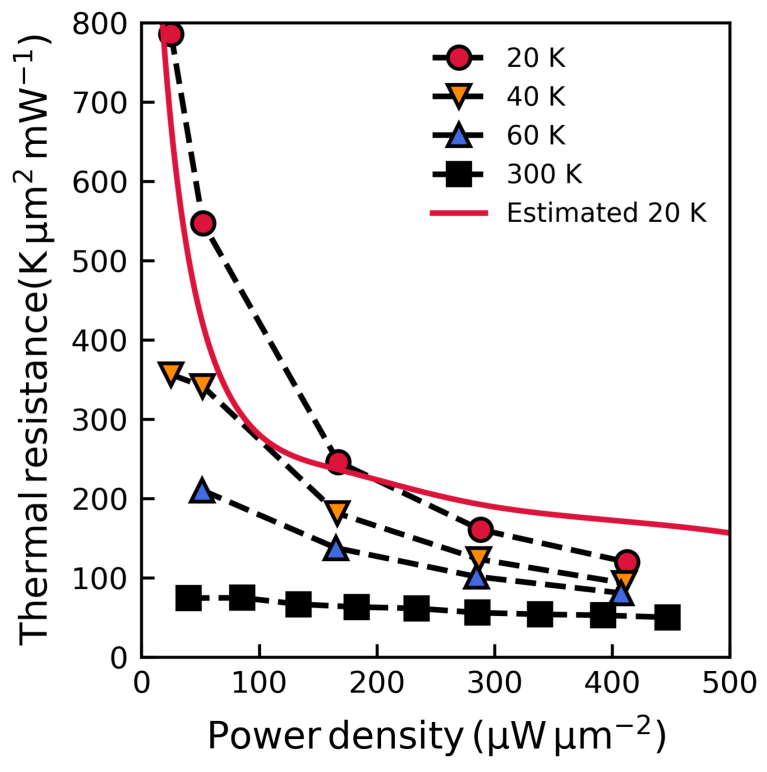

Figure 4: Thermal resistance of the junction versus power density at base temperatures $20 \mathrm{~K}$ (red circles), $40 \mathrm{~K}$ (yellow triangles), $60 \mathrm{~K}$ (blue triangles), and $300 \mathrm{~K}$ (black squares). At room temperature, the thermal resistance is nearly independent of power and thus junction temperature. At cryogenic temperatures, the thermal resistance increases nonlinearly as the power and junction temperature decrease. These features are qualitatively consistent with the predictions of a model assuming the thermal resistance is dominated by phonon radiation through an interface (computed at $20 \mathrm{~K}$, red solid line). Dashed black lines are added as guides to the eye.

$\mathrm{Pt}, \mathrm{Au}$, and subsequent bake and passivation steps induce atomic diffusion and the formation of intermetallic compounds [42]. As a result, knowledge of the atomic structure and vibrational modes of the interface required for such a model is lacking.

Considering these challenges, we instead construct a qualitative model for the thermal resistance in which the phonons are assumed to follow a Debye model and radiate from the gate through the interface. Assuming that the temperature is small compared to a Debye temperature associated with atomic vibrations at the interface, the heat flux through the interface obeys the StefanBoltzmann law $q=\epsilon \sigma_{p}\left(T_{j}^{4}-T^{4}\right)[45]$, where $\epsilon$ is a measure of the transmission of phonons through the interface and $\sigma_{p}=\pi^{2} k_{B}^{4} / 40 \hbar^{3} v_{\text {ave }}^{2} \sim 600 \mathrm{~W} \mathrm{~m}^{-2} \mathrm{~K}^{-4}$ is the Stefan-Boltzmann constant for phonons in GaAs. Here, $v_{a v e} \approx 3500 \mathrm{~m} \mathrm{~s}^{-1}$ is the Debye velocity in GaAs computed from the average sound velocities [40]. In this regime, the thermal resistance can be defined as $R_{t h}^{-1}=$ $\epsilon \sigma_{p}\left(T_{j}+T\right)\left(T_{j}^{2}+T^{2}\right)$.

Applying this model to the data measured at $20 \mathrm{~K}$, we obtain the curve shown in Fig. $2 \mathrm{~b}$. 
Despite the simplicity of the model, it qualitatively captures the nonlinear variation of thermal resistance with power for $\epsilon \sim 0.02$. We note that the thermal resistance predicted by diffusion theory based on the bulk thermal conductivity of GaAs at $T=20 \mathrm{~K}$ predicts a thermal resistance of $0.5 \mathrm{~K} \mathrm{\mu m}^{2} \mathrm{~mW}^{-1}$, orders of magnitude smaller than the observed resistance. The physical picture of heat dissipation from the gate that emerges is therefore the radiation of phonons from the gate with a heat flux that is smaller than the pure radiation value owing to phonon reflections at the gate-semiconductor interface.

\section{DISCUSSION}

We now discuss the implications of heat dissipation by phonon radiation on the self-heating and microwave noise figure of HEMTs. In the present experiments, the gate was forward-biased while the drain was grounded so that heat was generated by current flowing through the gate. However, under the typical low noise operating conditions for depletion-mode HEMTs, the gate and drain are reverse and forward biased, respectively, and heat is generated by current flowing through the channel. Despite these differences, the identification of the phonon radiation mechanism supported by the measurements in this study allows us to assess the magnitude of self-heating at the low-noise operating bias. Considering heat transport to occur by phonon radiation, at the low-noise bias phonons generated in the channel radiate to the gate which then radiates phonons to the substrate to balance the incoming heat flux. The steady-state temperature of the gate is set by the radiation space resistances between the gate, channel, and substrate.

An equivalent radiation circuit model can be used to predict the temperature rise in the gate from the radiative phonon flux originating at the drain (see Eqn. 6.48 of Ref. [46]). In this model, the three nodes in the circuit are the channel (c), gate (g), and substrate (s) linked by space radiation resistances. We assume all surfaces are black for simplicity. The gate node is adiabatic to excellent approximation so that all absorbed radiation is re-emitted (see Supplementary Information in Ref. [15]).

Under these assumptions, the gate temperature $T_{g}$ can be expressed as $J_{g}=\left(J_{d} R_{c g}+\right.$ $\left.J R_{g s}\right)\left(R_{c g}+R_{g s}\right)^{-1}$, where $R_{i j}=A_{i} F_{i j}$ is the space resistance between nodes $i$ and $j, A_{i}$ is the emitting line length, $F_{i j}$ is the view factor, and $J_{i}=\sigma_{p} T_{i}^{4}$ is the blackbody emissive power from node $i$ at temperature $T_{i}$. For a specified substrate temperature $T_{s}=T$ and power density from Joule heating at the channel $Q_{c}=\left(J_{c}-J_{g}\right) / R_{c g}+\left(J_{d}-J_{s}\right) / R_{c s}$, the radiation circuit can be solved for the channel and gate temperature rise. Based on typical HEMT geometry, we estimate 
emitting line lengths as $A_{g}=A_{c} \sim 70 \mathrm{~nm}$. The view factor is estimated from the intercept of the 2D solid angle of the gate from the emitter region in the channel. For a typical HEMT gemometry, we obtain $F_{c g} \sim 0.3$. Solving for the temperature rise, we find that the gate temperature rise is $\sim 2 / 3$ of that at the channel. At a typical low-noise bias, the dissipated power is $\sim 30 \mathrm{~mW} \mathrm{~mm}^{-1}$

[15]. Taking the base temperature $T=4 \mathrm{~K}$, the temperature rise in the channel is $\sim 25 \mathrm{~K}$ and the gate temperature is therefore predicted to exceed $20 \mathrm{~K}$.

The self-heating of the gate affects the microwave noise of HEMTs because the thermal noise associated with the gate resistance is added at the input. If the steady-state gate temperature exceeds the base temperature, the microwave noise will be larger than predicted based on the base temperature. The above analysis implies that the thermal noise contribution of the HEMT gate at liquid helium temperatures is several times larger than previously assumed. An incorrect gate temperature directly affects the extracted drain temperature and consequently the interpretation of the physical origin of noise in HEMTs. Finally, we note that previous work has interpreted noise saturation at liquid helium temperatures to the saturation of the drain noise added at the output (see Fig. 1d of Ref. [15]). Our measurements indicate that the observed noise saturation is in fact due to elevated thermal noise as the gate temperature saturates with base temperature, as seen in Fig. 3b.

This analysis indicates that self-heating will limit the minimum noise figure of HEMT amplifiers without decreases in power consumption or improvements in device thermal management that decrease the physical temperature of the gate. Recently, low noise amplifiers with power consumption of hundreds of $\mu \mathrm{W}$ were reported, a value that is several times lower than those of typical HEMTs [7]. While these reductions can reduce gate heating, the quartic dependence associated with phonon radiation means that even at $300 \mu \mathrm{W}$ and $T=4 \mathrm{~K}$, the gate temperature is predicted to be $\sim 10 \mathrm{~K}$. Therefore, additional considerations of thermal management are necessary to reduce the excess thermal noise resulting from self-heating. A possible strategy is using direct immersion in liquid helium, which is routinely used for thermal management of superconducting magnets used in high-energy physics experiments [47].

\section{SUMMARY}

We have presented measurements of the gate junction temperature and thermal resistance of a low-noise HEMT from cryogenic to room temperature obtained using a Schottky thermometry method. The magnitude and trend of the extracted thermal resistance versus power and base 
temperature are consistent with heat dissipation by phonon radiation through an interface. Considering phonon radiation as the dominant mechanism of heat transfer, we estimate the intrinsic temperature of the gate at the low-noise operating bias using a radiation circuit. The model predicts that at liquid helium temperatures, the gate will self-heat to a temperature several times that of the base temperature. Our measurements thus indicate that self-heating constitutes a practical lower limit for the minimum microwave noise figure of cryogenic HEMT amplifiers unless thermal management strategies to remove heat from the gate can be identified.

\section{ACKNOWLEDGMENTS}

A.Y.C., B.G. and A.J.M. were supported by the National Science Foundation under Grant No. 1911220. I.E. was supported by the National Science Foundation Graduate Research Fellowship under Grant No. DGE-1745301. Any opinions, findings, and conclusions or recommendations expressed in this material are those of the author and do not necessarily reflect the views of the

National Science Foundation. J.K. and A.J.M were supported by a Jet Propulsion Laboratory PDRDF 107614-20AW0099.

[1] E. W. Bryerton, M. Morgan, and M. W. Pospieszalski. Ultra low noise cryogenic amplifiers for radio astronomy. In 2013 IEEE Radio and Wireless Symposium, pages 358-360. IEEE, 2013. https:// ieexplore. ieee.org/document/6486740.

[2] M. W. Pospieszalski. Extremely low-noise amplification with cryogenic FETs and HFETs: 1970-2004. IEEE Microwave Magazine, 6(3):62-75, 2005. https://ieeexplore.ieee.org/document/1511915.

[3] J.J. Bautista, J.G. Bowen, N.E. Fernandez, Z. Fujiwara, J. Loreman, S. Petty, J.L. Prater, R. Grunbacher, R. Lai, M. Nishimoto, M.R. Murti, and J. Laskar. Cryogenic, x-band and ka-band inp hemt based lnas for the deep space network. In 2001 IEEE Aerospace Conference Proceedings (Cat. No.01TH8542), volume 2, pages 2/829-2/842 vol.2. IEEE, 2001. https://ieeexplore.ieee.org/ document/931264.

[4] P. Krantz, M. Kjaergaard, F. Yan, T. P. Orlando, S. Gustavsson, and W. D. Oliver. A quantum engineer's guide to superconducting qubits. Applied Physics Reviews, 6(2):021318, 2019. https:// doi.org/10.1063/1.5089550.

[5] J. M. Hornibrook, J. I. Colless, I. D. Conway Lamb, S. J. Pauka, H. Lu, A. C. Gossard, J. D. Watson, G. C. Gardner, S. Fallahi, M. J. Manfra, and D. J. Reilly. Cryogenic control architecture for large-scale quantum computing. Phys. Rev. Applied, 3:024010, Feb 2015. https://link.aps.org/doi/10.1103/ PhysRevApplied.3.024010. 
[6] J. Chow, J. Gambetta, E. Magesan, D. Abraham, A. Cross, B. Johnson, N. Masluk, C. Ryan, J. Smolin, S. Srinivasan, and M. Steffen. Implementing a strand of a scalable fault-tolerant quantum computing fabric. 5(4015), 2014. https://www.nature.com/articles/ncomms5015\#citeas.

[7] E. Cha, N. Wadefalk, G. Moschetti, A. Pourkabirian, J. Stenarson, and J. Grahn. A 300- $\mu$ W cryogenic HEMT LNA for quantum computing. In 2020 IEEE/MTT-S International Microwave Symposium (IMS), pages 1299-1302. IEEE, 2020. https://ieeexplore.iee.org/document/9223865.

[8] Frank Arute, Kunal Arya, Ryan Babbush, Dave Bacon, Joseph C. Bardin, Rami Barends, Rupak Biswas, Sergio Boixo, Fernando G. S. L. Brandao, David A. Buell, Brian Burkett, Yu Chen, Zijun Chen, Ben Chiaro, Roberto Collins, William Courtney, Andrew Dunsworth, Edward Farhi, Brooks Foxen, Austin Fowler, Craig Gidney, Marissa Giustina, Rob Graff, Keith Guerin, Steve Habegger, Matthew P. Harrigan, Michael J. Hartmann, Alan Ho, Markus Hoffmann, Trent Huang, Travis S. Humble, Sergei V. Isakov, Evan Jeffrey, Zhang Jiang, Dvir Kafri, Kostyantyn Kechedzhi, Julian Kelly, Paul V. Klimov, Sergey Knysh, Alexander Korotkov, Fedor Kostritsa, David Landhuis, Mike Lindmark, Erik Lucero, Dmitry Lyakh, Salvatore Mandrà, Jarrod R. McClean, Matthew McEwen, Anthony Megrant, Xiao Mi, Kristel Michielsen, Masoud Mohseni, Josh Mutus, Ofer Naaman, Matthew Neeley, Charles Neill, Murphy Yuezhen Niu, Eric Ostby, Andre Petukhov, John C. Platt, Chris Quintana, Eleanor G. Rieffel, Pedram Roushan, Nicholas C. Rubin, Daniel Sank, Kevin J. Satzinger, Vadim Smelyanskiy, Kevin J. Sung, Matthew D. Trevithick, Amit Vainsencher, Benjamin Villalonga, Theodore White, Z. Jamie Yao, Ping Yeh, Adam Zalcman, Hartmut Neven, and John M. Martinis. Quantum supremacy using a programmable superconducting processor. Nature, 574(7779):505-510, October 2019. https://doi. org/10.1038/s41586-019-1666-5.

[9] E. Cha, N. Wadefalk, P. Nilsson, J. Schleeh, G. Moschetti, A. Pourkabirian, S. Tuzi, and J. Grahn. 0.3-14 and 16-GHz wide-bandwidth cryogenic MMIC low-noise amplifiers. IEEE Transactions on Microwave Theory and Techniques, 66(11):4860-4869, 2018. https://ieeexplore.ieee.org/document/ 8500350 .

[10] Felix Heinz, Fabian Thome, Arnulf Leuther, and Oliver Ambacher. Noise performance of sub-100-nm metamorphic hemt technologies. In 2020 IEEE/MTT-S International Microwave Symposium (IMS), pages 293-296. IEEE, 2020. https://ieeexplore.ieee.org/abstract/document/9223783.

[11] A. H. Akgiray, S. Weinreb, R. Leblanc, M. Renvoise, P. Frijlink, R. Lai, and S. Sarkozy. Noise measurements of discrete hemt transistors and application to wideband very low-noise amplifiers. IEEE Transactions on Microwave Theory and Techniques, 61(9):3285-3297, 2013. https://ieeexplore. ieee.org/document/6566114/footnotes.

[12] M. Varonen, R. Reeves, P. Kangaslahti, L. Samoska, K. Cleary, R. Gawanade, A. Fung, T. Gaier, S. Weinreb, A.C. Readhead, S. Sarkozy, and R. Lai. A 75-116-GHz LNA with 23-K noise temperature at 108 GHz. Jet Propulsion Laboratory Technical Report, 2013. https://trs.jpl.nasa.gov/handle/ $2014 / 43236$. 
[13] David Cuadrado-Calle, Danielle George, Gary A. Fuller, Kieran Cleary, Lorene Samoska, Pekka Kangaslahti, Jacob W. Kooi, Mary Soria, Mikko Varonen, Richard Lai, and Xiaobing Mei. Broadband mmic lnas for alma band $2+3$ with noise temperature below $28 \mathrm{k}$. IEEE Transactions on Microwave Theory and Techniques, 65(5):1589-1597, 2017.

[14] M. W. Pospieszalski. Modeling of noise parameters of MESFETs and MODFETs and their frequency and temperature dependence. IEEE Transactions on Microwave Theory and Techniques, 37(9):13401350, 1989. https://ieeexplore.ieee.org/document/32217.

[15] J. Schleeh, J. Mateos, I. Íñiguez De-La-Torre, N. Wadefalk, P. A. Nilsson, J. Grahn, and A. J. Minnich. Phonon black-body radiation limit for heat dissipation in electronics. Nature Materials, 14(2):187-192, 2014. https://www. nature.com/articles/nmat4126.

[16] K. H. G. Duh, W. F. Kopp, P. Ho, P. . Chao, M. . Ko, P. M. Smith, J. M. Ballingall, J. J. Bautista, and G. G. Ortiz. 32-GHz cryogenically cooled HEMT low-noise amplifiers. IEEE Transactions on Electron Devices, 36(8):1528-1535, 1989. https://ieeexplore.ieee.org/document/30966.

[17] Mark A. McCulloch, Jan Grahn, Simon J. Melhuish, Per-Ake Nilsson, Lucio Piccirillo, Joel Schleeh, and Niklas Wadefalk. Dependence of noise temperature on physical temperature for cryogenic lownoise amplifiers. Journal of Astronomical Telescopes, Instruments, and Systems, 3(1):1 - 4, 2017. https://doi.org/10.1117/1. JATIS.3.1.014003.

[18] M. W. Pospieszalski. On the limits of noise performance of field effect transistors. In 2017 IEEE MTTS International Microwave Symposium (IMS), pages 1953-1956. IEEE, 2017. https://ieeexplore. ieee.org/abstract/document/8059045.

[19] S. Weinreb. Low-noise cooled GASFET amplifiers. IEEE Transactions on Microwave Theory and Techniques, 28(10):1041-1054, 1980. https://ieeexplore.iee. org/document/1130223.

[20] J. A. Mittereder, J. A. Roussos, W. T. Anderson, and D. E. Ioannou. Quantitative measurement of channel temperature of GaAs devices for reliable life-time prediction. IEEE Transactions on Reliability, 51(4):482-485, 2002. https : //ieeexplore . ieee .org/stamp/stamp. jsp?tp=\&arnumber $=1044347$.

[21] M Farzaneh, K Maize, D Lüerßen, J A Summers, P M Mayer, P E Raad, K P Pipe, A Shakouri, R J Ram, and Janice A Hudgings. CCD-based thermoreflectance microscopy: principles and applications. Journal of Physics D: Applied Physics, 42(14):143001, jun 2009. https://doi.org/10.1088/ 0022-3727/42/14/143001.

[22] M. Kuball, S. Rajasingam, A. Sarua, M. J. Uren, T. Martin, B. T. Hughes, K. P. Hilton, and R. S. Balmer. Measurement of temperature distribution in multifinger AlGaN/GaN heterostructure fieldeffect transistors using micro-Raman spectroscopy. Applied Physics Letters, 82(1):124-126, 2003. https://doi.org/10.1063/1.1534935.

[23] S. Choi, E. R. Heller, D. Dorsey, R. Vetury, and S. Graham. Thermometry of AlGaN/GaN HEMTs using multispectral Raman features. IEEE Transactions on Electron Devices, 60(6):1898-1904, 2013. https://ieeexplore.ieee.org/document/6507260. 
[24] Thomas Beechem, Samuel Graham, Sean P. Kearney, Leslie M. Phinney, and Justin R. Serrano. Invited article: Simultaneous mapping of temperature and stress in microdevices using micro-Raman spectroscopy. Review of Scientific Instruments, 78(6):061301, 2007. https://doi.org/10.1063/1. 2738946.

[25] Jeong Park, Moo Whan Shin, and C. C. Lee. Thermal modeling and measurement of GaN-based HFET devices. IEEE Electron Device Letters, 24(7):424-426, 2003. https://ieeexplore.ieee.org/ document/1217285.

[26] D. Takacs and J. Trager. Temperature increase by self-heating in VLSI CMOS. In ESSDERC '87: 17th European Solid State Device Research Conference, pages 729-732. IEEE, 1987. https://ieeexplore. ieee.org/abstract/document/5436746? casa_token=-6FuZz9KOiQAAAA : Oxc4K-qSvmi_4ako4pLP82vs0t9Yb76cV2btrk6uYe80uf9HyStcRXY15kw38bNMKQ3SB10qXQ.

[27] S. S. Sesnic and G. R. Craig. Thermal effects in JFET and MOSFET devices at cryogenic temperatures. IEEE Transactions on Electron Devices, 19(8):933-942, 1972. https://ieeexplore.ieee.org/ document/1476993.

[28] D. P. Foty and S. L. Titcomb. Thermal effects in n-channel enhancement MOSFETs operated at cryogenic temperatures. IEEE Transactions on Electron Devices, 34(1):107-113, 1987. https: //ieeexplore.ieee.org/document/1486603.

[29] D. Foty. Thermal effects in p-channel MOSFETs at low temperatures. IEEE Transactions on Electron Devices, 36(8):1542-1544, 1989. https://ieeexplore.ieee.org/document/30968.

[30] B. M. Tenbroek, M. S. L. Lee, W. Redman-White, J. T. Bunyan, and M. J. Uren. Self-heating effects in SOI MOSFETs and their measurement by small signal conductance techniques. IEEE Transactions on Electron Devices, 43(12):2240-2248, 1996. https://ieeexplore.ieee.org/document/544417.

[31] K. Triantopoulos, M. Cassé, S. Barraud, S. Haendler, E. Vincent, M. Vinet, F. Gaillard, and G. Ghibaudo. Self-heating effect in FDSOI transistors down to cryogenic operation at $4.2 \mathrm{~K}$. IEEE Transactions on Electron Devices, 66(8):3498-3505, 2019. https://ieeexplore. ieee.org/document/ 8741185.

[32] S García, I Íñiguez de-la Torre, J Mateos, T González, and S Pérez. Impact of substrate and thermal boundary resistance on the performance of AlGaN/GaN HEMTs analyzed by means of electrothermal Monte Carlo simulations. Semiconductor Science and Technology, 31(6):065005, apr 2016. URL https://doi.org/10.1088/0268-1242/31/6/065005. https://iopscience.iop.org/article/10. 1088/0268-1242/31/6/065005/meta.

[33] A. M. Darwish, A. J. Bayba, and H. A. Hung. Utilizing diode characteristics for GaN HEMT channel temperature prediction. IEEE Transactions on Microwave Theory and Techniques, 56(12):3188-3192, 2008. https://ieeexplore.ieee.org/abstract/document/4682665.

[34] M. Wu, X. Ma, L. Yang, Q. Zhu, M. Zhang, L. Yang, and Y. Hao. Accurate measurement of channel temperature for AlGaN/GaN HEMTs. IEEE Transactions on Electron Devices, 65(11):4792-4799, 2018. https://ieeexplore. ieee.org/document/8466809. 
[35] Bastian Mottet. Zuverlässigkeitsstudien an Höchstfrequenzbauelementen mit gepulsten Techniken (TLPMethode). PhD thesis, Technische Universität, 2005.

[36] T. Kiuru, J. Mallat, A. V. Raisanen, and T. Narhi. Schottky diode series resistance and thermal resistance extraction from $S$-parameter and temperature controlled I-V measurements. IEEE Transactions on Microwave Theory and Techniques, 59(8):2108-2116, 2011. https://ieeexplore.ieee. org/document/5773463.

[37] A. Akgiray. New Technologies Driving Decade-Bandwidth Radio Astronomy:Quad-Ridged Flared Horn 86 Compound-Semiconductor LNAs. PhD thesis, California Institute of Technology, Pasadena, CA, 2013.

[38] Damon Russell, Kieran Cleary, and Rodrigo Reeves. Cryogenic probe station for on-wafer characterization of electrical devices. Review of Scientific Instruments, 83(4):044703, 2012. https: //doi.org/10.1063/1.3700213.

[39] Roberto M. Cibils and Román H. Buitrago. Forward I-V plot for nonideal Schottky diodes with high series resistance. Journal of Applied Physics, 58(2):1075-1077, 1985. https://doi.org/10.1063/1. 336222.

[40] J. S. Blakemore. Semiconducting and other major properties of Gallium Arsenide. Journal of Applied Physics, 53(10):R123-R181, 1982. https://doi.org/10.1063/1.331665.

[41] R. J. Stoner and H. J. Maris. Kapitza conductance and heat flow between solids at temperatures from 50 to 300 K. Phys. Rev. B, 48:16373-16387, Dec 1993. https://link.aps.org/doi/10.1103/ PhysRevB.48.16373.

[42] E. Cha. InP High Electron Mobility Transistors for Cryogenic Low-Noise and Low-Power Amplifiers. PhD thesis, Chalmers University of Technology, Gothenburg, Sweden, 2020.

[43] P. Hopkins. Thermal transport across solid interfaces with nanoscale imperfections: Effects of roughness, disorder, dislocations, and bonding on thermal boundary conductance. International Scholarly Research Notices, 2013, 2013. https://www.hindawi.com/journals/isrn/2013/682586/.

[44] Chengyun Hua, Xiangwen Chen, Navaneetha K. Ravichandran, and Austin J. Minnich. Experimental metrology to obtain thermal phonon transmission coefficients at solid interfaces. Phys. Rev. B, 95: 205423, May 2017. https://link.aps.org/doi/10.1103/PhysRevB.95.205423.

[45] E. T. Swartz and R. O. Pohl. Thermal boundary resistance. Rev. Mod. Phys., 61:605-668, Jul 1989. https://link.aps.org/doi/10.1103/RevModPhys.61.605.

[46] A.F. Mills. Heat Transfer. Irwin, 1992. ISBN 9780256076424.

[47] Philippe Lebrun. Superfluid helium cryogenics for the large hadron collider project at CERN. Cryogenics, 34:1-8, January 1994. URL https://doi.org/10.1016/s0011-2275(05)80003-7. https: //www.sciencedirect.com/science/article/pii/S0011227505800037. 